Received: 18 January 2017

Accepted: 30 May 2017

Published online: 21 July 2017

\section{How cholesteryl ester transfer protein can also be a potential triglyceride transporter}

\author{
Venkat R. Chirasani \& Sanjib Senapati
}

CETP transfers cholesteryl esters (CEs) and triglycerides (TGs) between different lipoproteins and came in limelight as a drug-target against CVD. In the search for detailed mechanism of lipid transfer through CETP, enormous effort is devoted employing crystallographic, cryo-EM, and Molecular Dynamics (MD) studies. However, these studies primarily focused on CE-bound CETP structure and CE transfer mechanism. With the reported correlation that CETP looses significant CE transfer activity upon inhibiting TG transfer, it is of tremendous importance to understand the structure and dynamics of TGbound CETP. Our results from large-scale all-atom and coarse-grained MD simulations show that CETP can accommodate two TG molecules in parallel N-N orientation with TG oleate chains majorly attaining the tuning-fork conformation. In TG-bound form, CETP not only maintained its secondary structures but also exhibited similar bending-twisting motions as reported for CE-CETP crystal structure. Obtained structural information are further validated by correlating to available functional data of 2-8 fold slower transfer rate of TG through CETP, where we show that TGs make $20 \%$ additional contacts with CETP compared to CEs. Identified CETP residues facilitating TG binding also match very well with reported mutagenesis data. The study could accelerate the drug-designing processes to combat CETP functionality and CVD.

Cardiovascular disease (CVD) is one of the major causes of deaths in the world. Epidemiological analyses have identified multiple risk factors responsible for CVD. Out of these factors, high level of low-density lipoprotein (LDL), which supplies cholesterol in an uncontrolled manner to artery-wall macrophages was identified as a primary cause for the progression of CVD. On the other hand, elevated level of high-density lipoprotein (HDL) has been reported to inversely correlate to the progression of this disease $\mathrm{e}^{1-3}$. The ability of HDL to relocate cholesterol from macrophages to liver for excretion is vital in its cardio protective role ${ }^{4}$. Clinical studies on genetically modified mice with deficient HDL metabolic proteins conveyed compelling evidence that HDL is an important regulator of atherosclerosis in dyslipidemic conditions ${ }^{5}$. These findings have raised tremendous interest in utilizing HDL as a therapeutic target for the prevention of CVD.

In this context, cholesteryl ester transfer protein (CETP), a plasma glycol-protein with 476 residues came in the spotlight for its role in lipid metabolism and maintaining the HDL levels. In human blood plasma, CETP transports neutral lipids - cholesteryl esters (CEs) and triglycerides (TGs) between different lipoprotein fractions. In particular, CETP transfers CEs from HDL to LDL and VLDL (very low density lipoprotein) with the complementary transfer of TGs from LDL and VLDL to $\mathrm{HDL}^{6}$. It is seen that the hetero exchange of neutral lipids by CETP between atheroprotective HDL and atherogenic LDL or VLDL has an outcome of CE-depletion and TG-enrichment in HDL, which in turn catabolize HDL. Experimental studies on genetically CETP- deficient groups as well as clinical trials involving inhibition of $\mathrm{CETP}^{7}$ have provided conclusive evidence that CETP plays a crucial role in atherosclerosis. As increased activity or levels of CETP is inversely related to the HDL concentration in human blood plasma, inhibition of CETP through small molecule inhibitors is being pursued as an active approach to arrest $\mathrm{CVD}^{8-12}$. Some of the previously identified CETP inhibitors, including torcetrapib ${ }^{8}$ and dalcetrapib ${ }^{9}$ were discontinued due to their fatal side effects or futility in raising the HDL levels. However, new inhibitors like anacetrapib ${ }^{10}$ and BMS-79531 $1^{11}$ are currently in active clinical trials with marginal side effects in CVD patients. In spite of these developments, very little is known about the lipid transfer mechanism of CETP between HDL and LDLs ${ }^{13-18}$.

Bhupat and Jyoti Mehta School of Biosciences and Department of Biotechnology, Indian Institute of Technology Madras, Chennai, 600036, India. Correspondence and requests for materials should be addressed to S.S. (email: sanjibs@iitm.ac.in) 


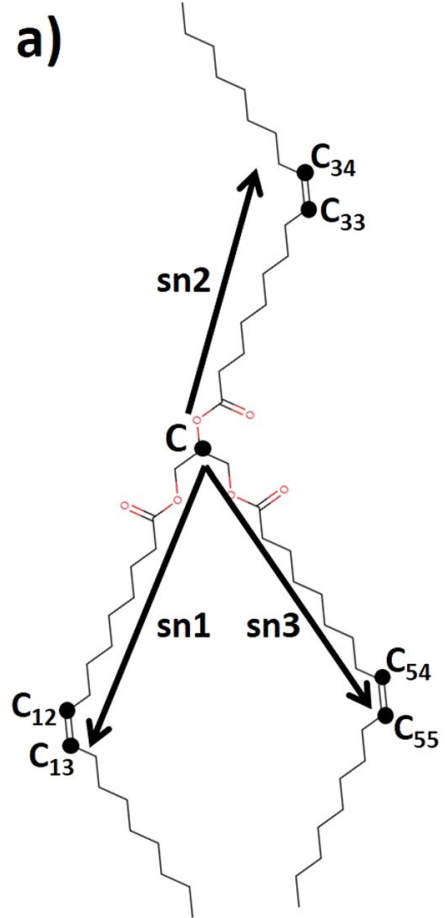

b)

Figure 1. (a) Molecular structure of a triolein molecule (TG). Three vectors - sn 1, sn2, sn 3 are defined on the molecular frame of TG to describe its different conformations. (b) Coarse grained representation of TG. Central glycerol bead is shown in rose color, carboxyl moiety-containing beads are shown in ochre, oleoyl apolar moieties are shown in cyan, and the unsaturated moieties are shown in pink bead.

The crystal structure of CETP (PDB ID: 2OBD) has been solved recently ${ }^{19}$. The structure shows a banana shaped protein with three important domains - N-terminal $\beta$-barrel domain, C-terminal $\beta$-barrel domain, and the central $\beta$-sheet domain. The crystal structure also shows a hydrophobic tunnel of length $60 \AA$ running through the central core of CETP and occupied by two cholesteryl esters (CEs) and two plug-in phospholipids (PLs). Interestingly, both this and available inhibitor bound CETP crystal structures ${ }^{20}$ are seen to be in CE-bound conformation. However, recent mutational studies have shown that CETP is also an active TG transporter, since the protein looses significant CE transfer activity upon the inhibition of TG transfer by active mutations in the hydrophobic tunnel ${ }^{19}$. A more direct evidence of the existence of TG bound CETP was reported in the pioneering work of Tall and coworkers from radiolabeled assays ${ }^{21}$. Unfortunately, till date there is no structural information available for the TG-bound CETP complex and a crystal structure is yet to be solved. These observations are insightful and thought provoking, suggesting a better comprehension of TG transfer mechanism of CETP is necessary. This prompted us to investigate the structure and dynamics of TG bound CETP, in this study.

Triglycerides, the tri-esters of glycerol are neutral lipids stored as lipid droplets in adipocytes. Two most common TGs present in human adipose tissues are tripalmitin and triolein. While tripalmitin is a saturated triglyceride with three palmitoyl chains attached to the glycerol moiety, triolein is an unsaturated triglyceride with three oleate chains connected to a central glycerol molecule (Fig. 1a). In blood plasma, TGs are transported by lipid transfer proteins, such as CETP for localizing to the core of the lipoproteins, particularly LDL and VLDL. Till today, very little is known about the structural conformations of TGs inside the lipid transfer proteins and lipoproteins. Molecular dynamics (MD) simulation study of the neat TGs (triolein) in liquid phase identified four major conformations of the triolein molecules - tuning fork, chair, trident, and random ${ }^{22}$. In the tuning fork and chair conformations, two of the three oleate chains reside on one side of the glycerol moiety and the third chain falls on the opposite side; in trident conformation, all the three oleate chains of TG reside on the same side of the glycerol moiety and thus point in the same direction; and in liquid or random conformation, the three oleate chains orient randomly. Out of these different conformations, TGs in tuning fork or chair conformation are most elongated. However, no such structural information of TGs bound to lipid transfer proteins, such as CETP is known.

The difficulty in investigating the conformational transitions and the mechanism of lipid transfer through CETP primarily arises due to the overall promptness of the processes. These processes occur in the time scale of nano- to micro-seconds, making it difficult to capture by available experimental techniques. In a recent study, we have unraveled the inherent plasticity in CETP structure that assisted CE transport through CETP by employing microsecond-long all-atom molecular dynamics (MD) simulations ${ }^{14}$. In this study, we attempt to propose a high-resolution model of TG bound CETP structure and decipher the mechanism of TG transport through CETP hydrophobic tunnel with the help of united-atom and coarse-grained MD simulations. We believe that this study would have important implications in CVD therapeutics targeting CETP functionality. 


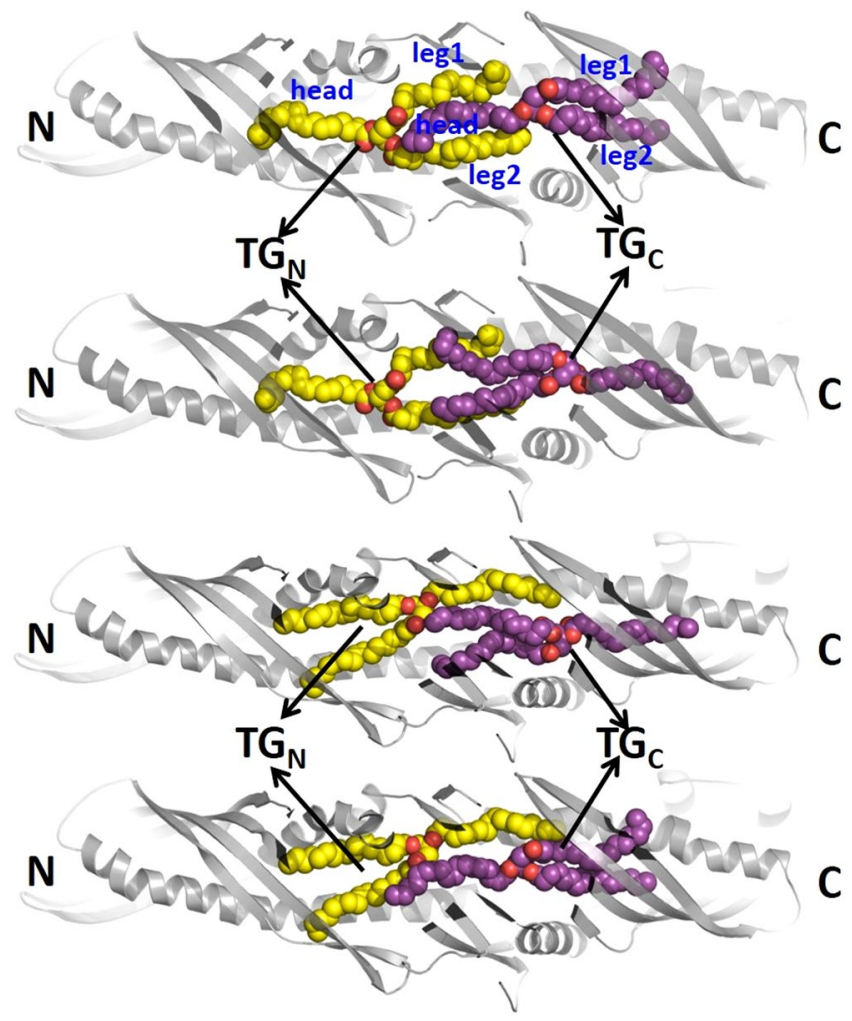

Figure 2. Possible orientations of two docked TG molecules in CETP core tunnel: (a) "parallel N-N" orientation with both TGs orienting head-to-legs from N- to C-terminal of CETP, (b) "antiparallel N-C" orientation with $\mathrm{N}$-terminal $\mathrm{TG}\left(\mathrm{TG}_{\mathrm{N}}\right)$ orienting head-to-legs from $\mathrm{N}$ - to C-terminal, and C-terminal TG $\left(\mathrm{TG}_{\mathrm{C}}\right.$ ) orienting head-to-legs from C- to N-terminal of CETP, (c) "parallel C-C" orientation with both TGs orienting head-to-legs from C- to N-terminal of CETP, (d) "antiparallel C-N" orientation with $\mathrm{TG}_{\mathrm{N}}$ orienting head-to-legs from $\mathrm{C}$ - to $\mathrm{N}$-terminal and $\mathrm{TG}_{\mathrm{C}}$ orienting head-to-legs from $\mathrm{N}$ - to C-terminal of CETP. $\mathrm{TG}_{\mathrm{N}}$ is shown in yellow and $T G_{C}$ in purple. Oxygen atoms in $T_{N}$ and $T G_{C}$ are shown in red. CETP is shown in gray cartoon.

\section{Results and Discussion}

The lack of structural information of TG-bound CETP has motivated us to explore TG conformation in CETP through MD simulations. Since the CE-bound CETP crystal structure contained two CE molecules and the structural lengths of CE and TG are similar ${ }^{19}$, we accommodated two TG molecules (triolein) in CETP core tunnel. Our protein-ligand docking results also show the formation of a tight complex between CETP and two TGs where the two TG molecules nicely fill up the $60 \AA$ CETP core tunnel. However, as we were uncertain about the orientations of TGs in CETP, we docked two TG molecules in CETP tunnel in all possible orientations. Fig. 2 depicts the four different possibilities:

a) System-I with both TGs orienting head-to-legs from N- to C-terminal of CETP; named as "parallel N-N" orientation of TGs.

b) System-II with $\mathrm{N}$-terminal $\mathrm{TG}\left(\mathrm{TG}_{\mathrm{N}}\right)$ orienting head-to-legs from $\mathrm{N}$ - to C-terminal and C-terminal $\mathrm{TG}$ $\left(\mathrm{TG}_{\mathrm{C}}\right.$ ) orienting head-to-legs from C- to N-terminal of CETP, named as "antiparallel N-C" orientation of TGs.

c) System-III with both TGs orienting head-to-legs from C- to N-terminal of CETP; named as "parallel C-C" orientation of TGs.

d) System-IV with $\mathrm{TG}_{\mathrm{N}}$ orienting head-to-legs from $\mathrm{C}$ - to $\mathrm{N}$-terminal and $\mathrm{TG}_{\mathrm{C}}$ orienting head-to-legs from $\mathrm{N}$ - to C-terminal of CETP; named as "antiparallel C-N" orientation of TGs.

It is worth mentioning here that we could accommodate only the tuning fork conformation of TGs in the narrow tunnel of CETP. This is because TGs in tuning fork or chair conformation are thinner and most elongated, while the TGs in trident and random conformations are wider and spread over a larger space (see the description of TG in "Introduction"). Also, since the length of CETP tunnel was only $60 \AA$, a significant overlap between the two TGs was unavoidable. Docking of TGs to CETP tunnel was performed manually by replacing CEs to TGs in the CETP crystal structure.

United atom simulations could not capture large-scale changes in TG orientations. The docked complexes of TG bound CETP were subsequently simulated in water for considerable amount of time (see 

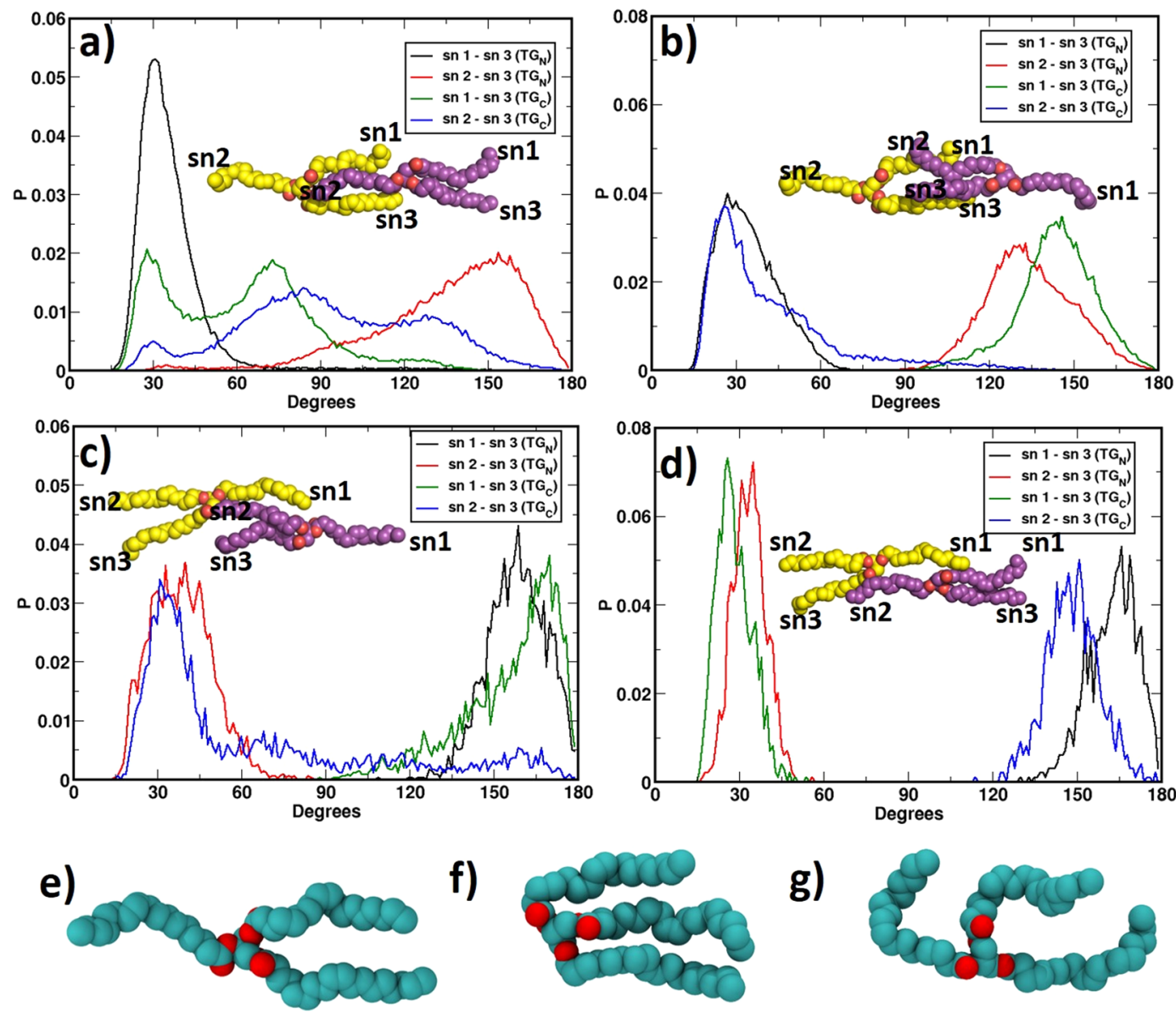

g)

Figure 3. Distribution of angles between the three oleate chains, sn 1 , sn 2 , sn 3 of both TGs from UA simulation data. Results are shown for bound TGs in CETP with (a) "parallel N-N" orientation, (b) "antiparallel N-C" orientation, (c) "parallel C-C" orientation, and (d) "antiparallel C-N" orientation. The color codes of the graphs are included in insets. Initial $\mathrm{TG}$ conformations $\left(\mathrm{TG}_{\mathrm{N}}\right.$ : yellow, $\mathrm{TG}_{\mathrm{C}}$ : purple) are shown for easy understanding. Different conformations of $\mathrm{TG}_{\mathrm{C}}$ observed during simulation of system-I are shown: (e) tuning fork, (f) trident, and (g) random.

Table S1). The visual inspection of simulation trajectories shows significant conformational flexibility of the TGs in CETP tunnel. To quantify the conformational changes, we first defined suitable vector on each oleate chain in TG. The vectors were constructed originating from the central carbon, $C$, of the TG molecule to the centre of mass of the $\mathrm{C}=\mathrm{C}$ double bond in each oleate chain as shown in Fig. 1a (the latter point also represents the COM of the whole oleate chain). These three vectors are named as: sn1, sn2, and sn3. Subsequently, we measured the angles subtended by these vectors during the entire course of simulations. Fig. 3 presents the distribution of angles in all four systems depicting the TG conformations in CETP. For the convenience of calculations, we always termed the oleate chain pointing toward the $\mathrm{N}$-domain as sn2, the one pointing toward the $\mathrm{C}$-domain as sn1, and the third one that moves either way is termed as sn3, for both the TGs (Fig. 3 insets).

As Fig. 3a shows, the N-terminal TG in system-I exhibits a sharp peak at $30^{\circ}$ for the sn 1 -sn 3 distributions and a distinct peak at $150^{\circ}$ for the sn2-sn 3 distributions, suggesting that $\mathrm{TG}_{\mathrm{N}}$ maintained the original tuning fork conformation (Fig. 3e) with its head-to-legs pointing from $\mathrm{N}$ - to $\mathrm{C}$-terminal of CETP. The C-terminal TG, on the other hand, exhibits interesting conformational changes. The sn1-sn3 angle displays a bimodal distribution with major peaks at $30^{\circ}$ and $75^{\circ}$, and sn $2-s n 3$ distribution exhibits three peaks at $30^{\circ}, 85^{\circ}$, and $135^{\circ}$. The sn1-sn3 peak at $30^{\circ}$ and sn 2 -sn 3 peak at $135^{\circ}$ signify the original tuning fork conformation (Fig. $3 \mathrm{e}$ ) of the $\mathrm{TG}_{\mathrm{C}}$ with head-to-legs pointing from $\mathrm{N}$ - to C-terminal of CETP. On the other hand, both sn1-sn3 and sn2-sn3 peaks at $30^{\circ}$ correspond to the existence of $\mathrm{TG}_{\mathrm{C}}$ in trident conformation (Fig. $3 \mathrm{f}$ ), and the respective peak at $75^{\circ}$ and $85^{\circ}$ represent a random conformation of $\mathrm{TG}_{\mathrm{C}}$ (Fig. $3 \mathrm{~g}$ ). Thus, the distributions of $\mathrm{TG}$ oleate angles suggest that two TG molecules in CETP tunnel majorly reside in the "parallel N-N" orientation, even though the C-terminal TG explores various other conformations. The restricted dynamics of $\mathrm{TG}_{\mathrm{N}}$ and relaxed nature of $\mathrm{TG}_{\mathrm{C}}$ are in accordance with the architecture of CETP, which shows narrower versus relatively wider tunnel in $\mathrm{N}$-versus $\mathrm{C}$-terminal domain of the protein in CE-bound crystal structure ${ }^{19}$.

System-II with initial "antiparallel N-C" orientation of the two TGs displayed minor changes in TG conformations (Fig. 3b). $\mathrm{TG}_{\mathrm{N}}$ remained stable in its original tuning fork conformation with sn1-sn3 and sn2-sn3 

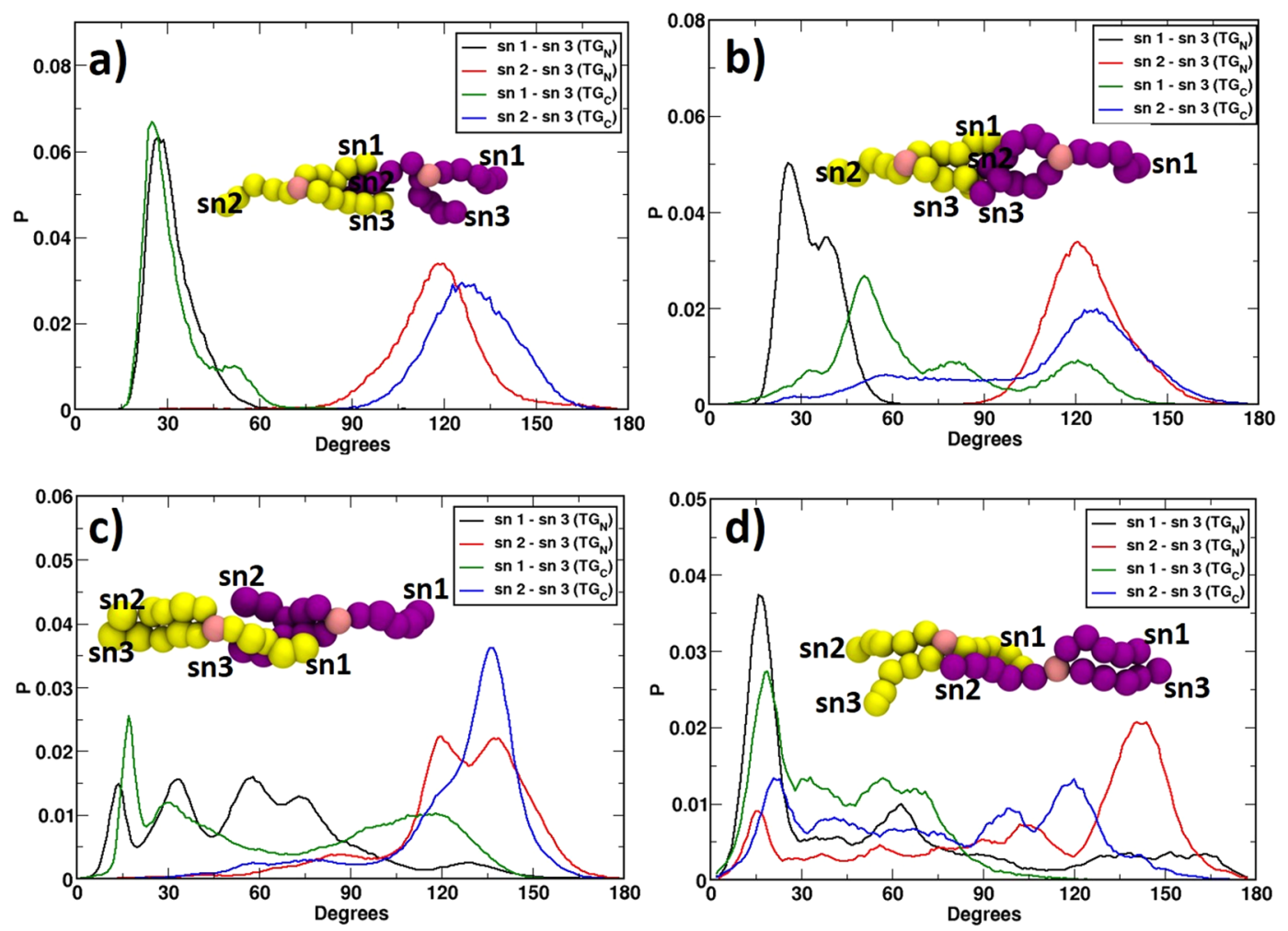

Figure 4. Distribution of angles between the three oleate chains, sn 1, sn2, sn 3 of both TGs from CG simulation data. Results are shown for bound TGs in CETP with (a) "parallel N-N" orientation, (b) "antiparallel N-C" orientation, (c) "parallel C-C" orientation, and (d) "antiparallel C-N" orientation. The color codes of the graphs are included in insets. Initial TG conformations $\left(\mathrm{TG}_{\mathrm{N}}\right.$ : yellow, $\mathrm{TG}_{\mathrm{C}}$ : purple) are shown for easy understanding.

angles distributed majorly at $30^{\circ}$ and $135^{\circ}$, respectively. On the other hand, $\mathrm{TG}_{\mathrm{C}}$ shows the sn $1-\mathrm{sn} 3$ peak at $145^{\circ}$ and sn 2 -sn 3 peak at $30^{\circ}$, which also correspond to its original antiparallel orientation. However, the broad distribution of the sn2-sn 3 angle even up to $120^{\circ}$ implies that $\mathrm{TG}_{\mathrm{C}}$ is not very stable in the antiparallel orientation and has a tendency to revert. A very similar situation prevailed in system-III with both TGs initially orienting head-to-legs from $\mathrm{C}$ - to $\mathrm{N}$-terminal of CETP displayed an unstable $\mathrm{TG}_{\mathrm{C}}$, even though the $\mathrm{TG}_{\mathrm{N}}$ conformation remained unchanged (Fig. $3 \mathrm{c}$ ). Thus $\mathrm{TG}_{\mathrm{N}}$ was confined in the initial legs-to-head orientation with single major peak for both sn1-sn3 and sn2-sn3 distributions. But, $\mathrm{TG}_{\mathrm{C}}$ exhibited a broad spectrum of distribution for both the angles with sn2-sn 3 angle extending up to $170^{\circ}$ from its initial value of $30^{\circ}$. This again implies that $\mathrm{TG}_{\mathrm{C}}$ in legs-to-head orientation from N- to C-terminal of CETP is misfitting. The angle distribution in system-IV with "antiparallel C-N" orientation of TGs exhibits the least conformational changes (Fig. 3d). Interestingly, unlike systems II and III, here TG $_{\mathrm{C}}$ in the head-to-legs orientation remained extremely stable with very narrow distributions for both sn1-sn3 and sn2-sn3 angles, suggesting that the head-to-legs orientation from $\mathrm{N}$ - to C-terminal of CETP could be the preferred conformation of $\mathrm{TG}_{\mathrm{C}}$.

The narrow distribution of $\mathrm{TG}_{\mathrm{N}}$ angles, particularly in systems III and IV, warrants a discussion. If we presume that head-to-legs is the preferred orientation of $\mathrm{TG}_{\mathrm{N}}$ (Fig. 3a and b), this lipid should have sampled a wide range of conformations in systems III and IV showing its tendency to flip. However, no such transitions took place. When we looked back the architecture of CETP tunnel in the crystal structure, we found that N-terminal region of CETP tunnel is narrower than the $\mathrm{C}$-terminal region ${ }^{19}$. Thus the packing of two parallel oleate chains $\left(\mathrm{TG}_{\mathrm{N}}\right.$ legs in legs-to-head orientation) in this constricted region leaves little space for $\mathrm{TG}_{\mathrm{N}}$ to sample new conformations in systems III and IV. This prompted us to employ coarse-grained molecular dynamics simulations to unravel the TG dynamics at larger timescales.

CG simulations depict "parallel N-N" as the preferred orientation of the bound TGs in CETP. United atom simulations revealed limited degrees of freedom of bound TGs in CETP tunnel due to insufficient sampling. Hence, we transformed the united atom systems to coarse-grained representation. We employed ElNeDyN forcefield to keep the secondary structure of CETP intact and simulated each of the four systems for long enough time till the TG orientations were converged (Table S1). Again, we quantified the conformational changes in TG molecules by defining three vectors along the oleate chains. These vectors were drawn from TG's central bead GLY to the double bond defining beads D2A, D2B, and D2C and named as sn1, sn2, and sn3, respectively (see Fig. 1b). Figure 4 depicts the TG angle distributions in four CG systems. As before, we again termed the oleate chain pointing toward the $\mathrm{N}$-domain as sn2, the one pointing toward the $\mathrm{C}$-domain as sn1, and the third one that moves either way as sn3, for both the TGs. 
As Fig. 4a shows, the distributions of both sn1-sn3 and sn2-sn3 angles of $\mathrm{TG}_{\mathrm{N}}$ in system-I are very sharp with respective single peak at $30^{\circ}$ and $120^{\circ}$, representing $\mathrm{TG}_{\mathrm{N}}$ 's stability in the initial tuning fork conformation with head-to-legs orientation from N- to C-terminal of CETP. For $\mathrm{TG}_{\mathrm{C}}$ also a similar stability was observed in the original tuning fork conformation with sn1-sn 3 and sn2-sn 3 distributions displaying major peaks at $30^{\circ}$ and $130^{\circ}$, respectively. Such a distribution of the two TG molecules signifies their preferred "parallel N-N" orientation in CETP tunnel. In system-II with TGs initially in antiparallel N-C orientation (Fig. 4b), the distribution of sn1-sn3 angle of $\mathrm{TG}_{\mathrm{N}}$ showed a major peak at $25^{\circ}$ with a small shoulder at $40^{\circ}$, while the sn2-sn 3 distribution showed a single major peak at $120^{\circ}$. This inter-oleate distribution therefore signifies a stable head-to-legs orientation of $\mathrm{TG}_{\mathrm{N}}$ from $\mathrm{N}$ - to $\mathrm{C}$-terminal of CETP. The angle distribution of $\mathrm{TG}_{\mathrm{C}}$ shows a few interesting features. The broad distributions of its both sn1-sn3 and sn2-sn3 angles imply major conformational rearrangements. The sn1-sn3 distribution showed a major peak at $50^{\circ}$ and two minor peaks at $80^{\circ}$ and $120^{\circ}$. The peak at $120^{\circ}$ corresponds to its initial legs-to-head orientation from $\mathrm{N}$ - to C-terminal of CETP. However, the major peak at $50^{\circ}$ implies the reversal of sn 3 orientation from the N-terminal side of CETP to the C-terminal side. In accordance, the distribution of sn2-sn 3 angles exhibits a major peak at $125^{\circ}$ and a broad peak at $55^{\circ}$. The peak at $55^{\circ}$ denotes the initial orientation, where the sn 2 and sn 3 chains were together in the $\mathrm{N}$-terminal side of CETP. But the major peak at $125^{\circ}$ again indicates the switching of sn 3 from N-terminal to C-terminal side of CETP. To confirm that this re-distribution is due to the switching of sn 3 chain and not for the interchange of $\mathrm{sn} 1$ and sn 2 chains, we have done a thorough visual inspection of the simulation trajectory and the corresponding movie is presented in SI (Movies M1-M4). Thus the major sn 1 -sn 3 peak at $50^{\circ}$ and $\operatorname{sn} 2$-sn 3 peak at $125^{\circ}$ suggest the emergence of the tuning fork conformation of $\mathrm{TG}_{\mathrm{C}}$ in head-to-legs orientation from $\mathrm{N}$ - to C-terminal of CETP as the major conformation. The sn1-sn3 minor peak at $80^{\circ}$ along with the broad distribution of sn2-sn3 angle around a similar value imply the occurrence of the random configuration of $\mathrm{TG}_{\mathrm{C}}$ as the intermediate conformation during this orientational transition.

In system-III with both TGs initially in legs-to-head orientation from N- to C-terminal of CETP, the conformational changes appear to be even more drastic (Fig. 4c). The sn1-sn3 distribution of $\mathrm{TG}_{\mathrm{N}}$ exhibits a dual peak around $30^{\circ}$, another dual peak around $70^{\circ}$, and a minor peak at $130^{\circ}$. The sn2-sn 3 distribution shows sharp dual peaks at $130^{\circ}$, a small flat peak around $80^{\circ}$, and a minute population at smaller angles. The minute population of sn2-sn 3 at smaller angles and a very small sn1-sn 3 peak at $130^{\circ}$ imply that the initial conformation of $\mathrm{TG}_{\mathrm{N}}$ has almost vanished and the lipid transformed to new configurations. Thus, the major sn1-sn 3 distribution around $30^{\circ}$ along with sn 2 -sn 3 distribution at $130^{\circ}$ suggests the reversal of sn 3 from $\mathrm{N}$ - to C-terminal side of CETP with the appearance of head-to-legs orientation of $\mathrm{TG}_{\mathrm{N}}$. The dual sn 1 -sn 3 peak around $70^{\circ}$ along with flat $\operatorname{sn} 2$-sn3 peak at $80^{\circ}$ represent the intermediate random conformation during this transition of the lipid. Similarly, $\mathrm{TG}_{\mathrm{C}}$ also shows sharper distributions of both sn $1-\operatorname{sn} 3$ and sn $2-\operatorname{sn} 3$ angles at $20^{\circ}$ and $130^{\circ}$, respectively, representing the reversal of sn 3 chain for the formation of head-to-legs orientation from the initial legs-to-head orientation that show very small population (represented by minute sn 2 -sn 3 populations at $<50^{\circ}$ and minor peak of sn 1 -sn 3 at $120^{\circ}$ ) during the $16 \mu$ s long CG simulation. Taken together, the parallel C-C orientations of the bound TGs in CETP tunnel also transform to the "parallel N-N" orientation, very similar to system-II.

System-IV with TGs initially in "antiparallel C-N" orientation also converged to "parallel N-N" orientation by reversing the orientation of $\mathrm{TG}_{\mathrm{N}}$, while maintaining $\mathrm{TG}_{\mathrm{C}}$ in initial head-to-legs orientation from $\mathrm{N}$ - to C-terminal of CETP (Fig. $4 \mathrm{~d}$ ). The reversal of $\mathrm{TG}_{\mathrm{N}}$ orientation is evident from the appearance of the major acute angle peak in sn1-sn 3 distribution at around $15^{\circ}$ along with the obtuse angle peak in sn2-sn 3 distribution at $142^{\circ}$. Interestingly, both $\mathrm{TG}_{\mathrm{N}}$ and $\mathrm{TG}_{\mathrm{C}}$ have intermittently visited trident conformation in this system, which is apparent from the broad distributions of their both sn 1 -sn 3 and sn 2 -sn 3 angles between $15^{\circ}$ and $40^{\circ}$. Nonetheless, the turning fork remains the major conformation of both TGs as implied by their major peaks at requisite angles. The non-appearance of a trident conformation of $\mathrm{TG}_{\mathrm{C}}$ in $\mathrm{CG}$ simulation of system-I is presumably due to the structural complementarity of bound TG with CETP tunnel at CG level and, therefore, a quick stabilization. In system-IV, this could be observed due to the initial instability in $\mathrm{TG}_{\mathrm{N}}$. Thus the CG simulations of TG bound CETP systems with different inter-TG orientations converged to "parallel N-N" orientations of two bound TGs inside CETP hydrophobic tunnel.

To quantify the extent of different conformations of the TG pairs in CETP tunnel, we further calculated the probability distributions of $\mathrm{TG}_{\mathrm{N}}-\mathrm{TG}_{\mathrm{C}}$ orientations in all four systems from CG simulation data. With our prescribed definition of the oleate chain pointing toward the $\mathrm{N}$-domain as sn2 and the one pointing toward the $\mathrm{C}$-domain as sn 1 , the determination of sn1-sn 3 angle alone turned out to be the sufficient metric to trace the orientation of each TG. Thus for both TGs, if sn1-sn3 angle is less than $75^{\circ}$ then the lipid is resting in head-to-legs orientation from $\mathrm{N}$ - to C-terminal of CETP, for sn1-sn3 angle between $105^{\circ}$ and $180^{\circ}$ it is resting in legs-to head orientation from $\mathrm{N}$ - to $\mathrm{C}$-terminal of CETP, and for the intermediate angles it is resting in random orientation. With these criteria, the computed probability distributions of the orientations of TG pairs in all four systems are shown in Fig. 5. It is evident from this figure that system-I retains the original "parallel N-N" orientations of the TG pairs with $100 \%$ probability. System-II retains only $19.9 \%$ of the original "antiparallel N-C" orientation and produces significantly high $64.6 \%$ "parallel N-N" orientations after exploring $15.5 \%$ random conformations of the TG pairs during the conformational transition. Similarly, starting with the "parallel C-C" orientation, system-III exhibits $37.9 \%$ "parallel N-N", only $1 \%$ original "parallel C-C", and the remaining $61.1 \%$ intermediate conformations that constitute 4.7\% "antiparallel C-N", 22.1\% "antiparallel N-C", and 34.3\% random orientations of the TG pairs. System-IV with initial "antiparallel C-N" orientations displayed $67.1 \%$ "parallel N-N", $16.3 \%$ original "antiparallel C-N", $0.3 \%$ of "antiparallel N-C", $0.1 \%$ "parallel C-C", and the remaining $16.2 \%$ random orientations of the CETP bound TG pairs. A few interesting features can be noted from these distributions. Systems III and $\mathrm{IV}$, where $\mathrm{TG}_{\mathrm{N}}$ was initially in legs-to-head orientation, produced "parallel N-N" orientation in a lesser extent compared to system-II, which also induced significant conformational changes to its bound TGs. This can be attributed to the tunnel architecture of CETP, which is narrow in N-domain and therefore restricted the conformational sampling of $\mathrm{TG}_{\mathrm{N}}$ whose legs were tightly stacked in this narrow $\mathrm{N}$-terminal region of CETP. Also as one 


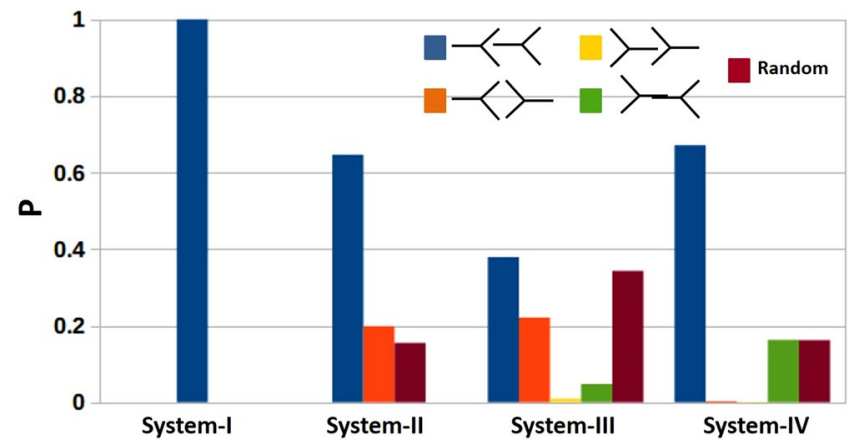

Figure 5. Probability distributions of the orientations of $\mathrm{TG}_{\mathrm{N}}-\mathrm{TG}_{\mathrm{C}}$ pairs in all CG systems from simulation data. Color code: BLUE: "parallel N-N", ORANGE: "antiparallel N-C", YELLOW: "parallel C-C", GREEN: “antiparallel C-N", BROWN: random orientations of TG pairs bound to CETP.

would expect, system III, which required to switch both the TG orientations, explored largest number of random and other intermediate conformations compared to other three systems to bring about the changes.

To strengthen our findings of preferred "parallel N-N" orientation of the bound TGs in CETP, we performed a second set of UA and CG simulations for each system. Each replica UA system was simulated for $500 \mathrm{~ns}-600 \mathrm{~ns}$ and each replica CG system was simulated for $10 \mu$ s. These replica simulations were started with completely different initial velocities as assigned randomly from a Maxwell-Boltzmann distribution. The details of these systems are included in Table S1. The calculated angle distributions for replica systems I-IV from UA simulations are presented in Fig. S1 and that for the replica systems I-IV from CG simulations are presented in Fig. S2. Similar to the primary set of simulations, the UA replica simulations could not capture large-scale changes in TG orientations (see Fig. 3 and Fig. S1). However, the replica CG simulations demonstrate that, regardless of the initial conformations and velocities, the pair of TGs in CETP core tunnel converges to "parallel N-N" orientation (see Fig. 4 and Fig. S2).

Thus from the UA and CG simulations, we found that TGs in CETP prefer to be in "parallel N-N" orientation. This finding can be justified by the following facts. CETP transfers CEs from HDL to LDL/VLDL and TGs from LDL/VLDL to $\mathrm{HDL}^{6}$. Recent polyclonal antibody tests in combination with EM study have suggested that CETP penetrates its N-terminal end into HDL and the C-terminal end into LDL/VLDL for lipid exchange ${ }^{13}$. This implies that TGs enter into the CETP tunnel (from LDL/VLDL) predominantly through the C-terminal end of CETP. Moreover, since entering the narrow CETP tunnel by inserting only one of the three identical oleate chains would require a smaller energy barrier to cross, TGs enter CETP in head-to-leg conformation with the single oleate chain (head) pointing toward the N-domain and the other two chains (legs) pointing toward C-domain. Moreover, since the tunnel in N-domain of CETP is narrower, after entering in the head-to-legs conformation, $\mathrm{TG}_{\mathrm{N}}$ remains stable in such form for majority of the time. Though the wider $\mathrm{C}$-domain allows $\mathrm{TG}_{\mathrm{C}}$ to undergo certain degree of conformational changes, this TG also prefers the head-to-legs conformation, as the CETP central domain is not wide enough to accommodate four oleate chains. Notably, both the CEs in CETP crystal structure, which might have entered through the N-terminal end of CETP from HDL, are oriented similarly with the long acyl chain (oleate chain) of both CEs pointing toward the C-terminal of CETP ${ }^{19}$. After finding the "parallel $\mathrm{N}-\mathrm{N}$ " as the major conformation of the bound TGs in CETP, we then wanted to check their influence on the structural stability of CETP and to compare the TG-bound CETP structure with the available crystal structure of CE-bound CETP. Hence, we resume our analyses on the primary $600 \mathrm{~ns}$ united-atom simulation data of CETP complexed with TGs in parallel N-N orientation (i.e. system-I in Table S1), and the results are presented below.

Secondary structural elements of CETP remain stable in TG bound complexes. The stability of secondary structural elements in a protein is crucial for its proper functioning. Hence it is important to examine the structural stability of CETP during the conformational transitions of its bound TGs. A simple strategy is to examine the mean squared displacements of the protein residues from their initial positions. Hence, we plotted the time evolution of root mean squared displacements (RMSD) of the C $\alpha$ atoms of CETP with respect to the available CE-bound CETP crystal structure (Fig. S3). For comparison, we have also simulated the CE-bound CETP crystal structure as the control and the corresponding RMSD profile is included. The figure shows the convergence of RMSD at around $150 \mathrm{~ns}$ of simulation time for both the systems. However, TG bound CETP exhibited higher RMSD compared to the CE-CETP complex. Such a trend is not unexpected considering the bulkier size and shape of TGs and, therefore, the larger dynamics of CETP to accommodate these bulky molecules optimally. Hence, the subsequent analyses on CETP were performed on the simulation data between 150-600 ns.

The stability of the protein is also examined by analyzing its secondary and tertiary structures. As shown in Fig. S4, the total number of residues that constitute different secondary structural elements of the protein remained unchanged throughout the simulation, suggesting no significant disruption of the secondary structure. To validate this further, we have performed the similar analysis on the control system, i.e. the CE-bound CETP crystal structure. As Fig. S4a,b show, the protein maintained its structural stability in both complexes with equal propensity. More specifically, the persistence of $\alpha$-helices and $\beta$-sheets throughout the simulation trajectory suggests that no significant transition had taken place among CETP secondary structural elements in the TG-bound complex. Analysis of secondary structure was performed by GROMACS utility program do_dssp, which assigns 

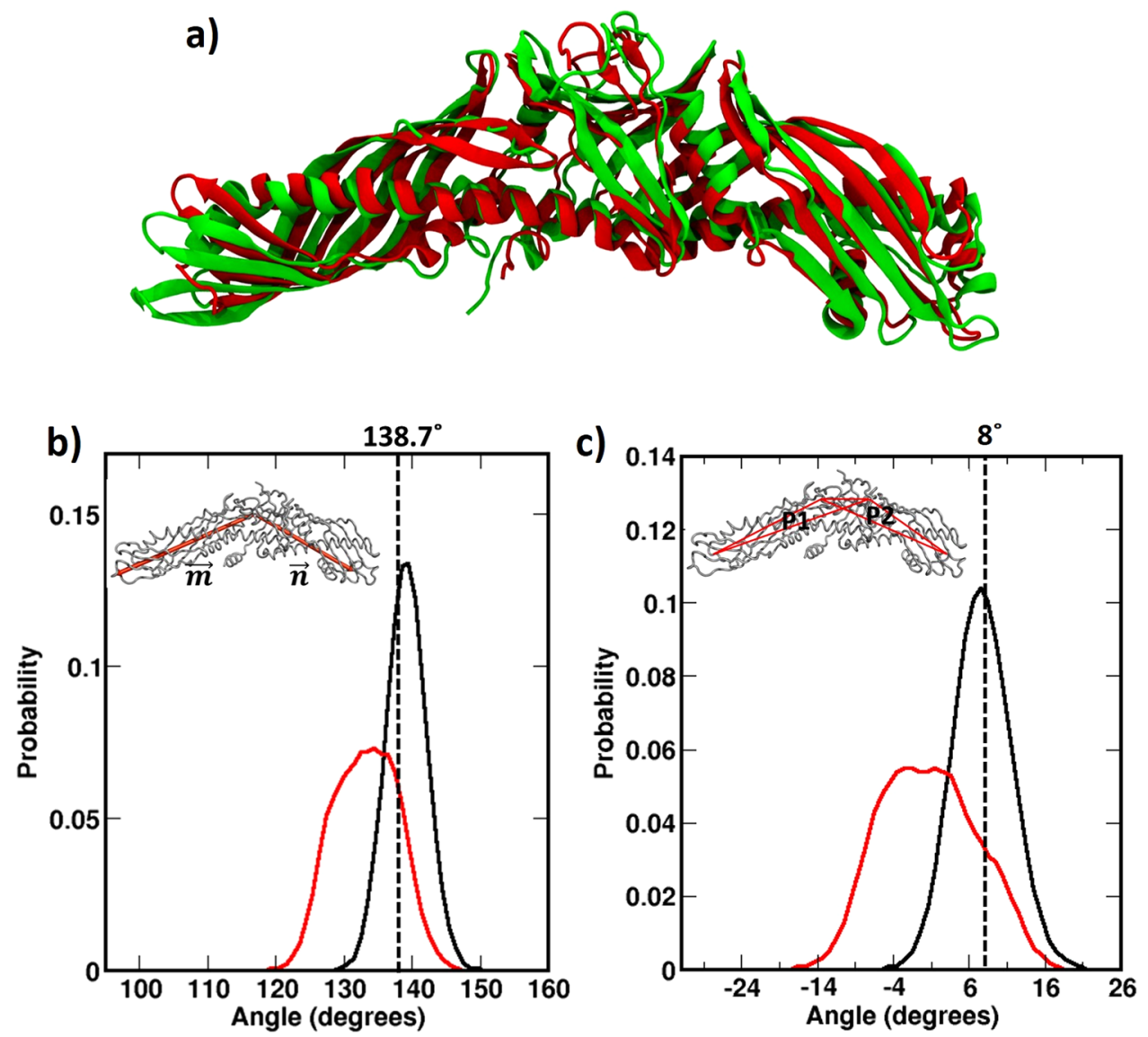

Figure 6. Comparison of TG- and CE-bound CETP structures. (a) Superposition of average structures of TG bound (green) and CE bound CETP (red) obtained from respective simulation trajectories. (b) Probability distribution of bending motion exhibited by CETP when bound to TG (red) and CE (black) during UA simulations. Bending was estimated from the angle between two vectors, and as shown in the inset. The dotted line indicates the angle of bending observed in CE-CETP crystal structure. (c) Probability distribution of twisting/untwisting motion exhibited by CETP when bound to TG (red) and CE (black) during UA simulations. Twisting was computed by measuring the angle between two planes, P1 and P2 as shown in the inset. The twist angle observed in CE-CETP crystal structure is shown in dotted line for reference.

secondary structure by analysing hydrogen bonding patterns among the protein residues. Thus, although there were significant changes in TG conformations in the core of CETP, the protein maintained its secondary structures throughout the simulation. A similar stability was also observed in the replica simulation as shown in Fig. S5.

To compare the tertiary structures of TG-bound and CE-bound CETP, we generated the average structure of the protein from the respective simulation trajectory (from last $20 \mathrm{~ns}$ data) and superposed. Results are shown in Fig. 6a. The superposition showed not so significant structural deviation with a RMSD of $2.67 \AA$ between the two structures. The local alignment produced even smaller deviations with the important structural units, viz. $\mathrm{N}$-terminal $\beta$-barrel $(0.468 \AA)$, central $\beta$-sheet $(0.255 \AA)$, C-terminal $\beta$-barrel (1.27 $\AA$ ) showing minimal changes. We noted that the larger RMSD from the whole structure superposition arises from an untwisting motion of $\mathrm{N}$-terminal tip in TG-bound complex, as seen in Fig. 6a. We will elaborate on this in the next section. We also examined the similarity in the binding location of the bound phospholipids (PLs) in TG-bound and CE-bound CETP structures, since PLs are vital in the structural integrity of CETP, in protecting the hydrophobic tunnel from the polar solvent, and in inhibitor entry ${ }^{20}$. The structural alignment showed close resemblance with a RMSD of 1.32 A. Furthermore, we examined the time evolution of CETP core tunnel in TG-bound CETP structure. Very interestingly, as Fig. S6 shows, the CETP tunnel significantly elongates to an extended length of $\sim 100 \AA$ A. However, we have also observed that the hydrophobic tunnel frequently constricts to $\sim 75 \AA$. The subsequent visual inspection of the trajectory demonstrates that the frequent inter-switching of $\mathrm{TG}_{\mathrm{C}}$ between the chair and trident/random conformations is the possible reason for the variation in CETP tunnel length. The extended tunnel observed in the current study is consistent with our recent report on the structural plasticity of CE-bound CETP, where we have shown that CE-bound CETP frequently switches to an extended conformation of about $100 \AA \AA$ in length ${ }^{14}$. Thus all these analyses suggest that TG-bound CETP maintains a similar stable conformation as that of the CE bound CETP. 
TG bound CETP exhibits similar dynamics as that of the CE-CETP crystal structure. To investigate the effects of bound TGs on the dynamics of CETP, we examined the angular motions - the bending and twisting of CETP during the course of the simulations. Visual inspection revealed a continuous harmonic motion with CETP swinging between the bent and stretched conformations. We termed this unique motion of CETP as the "breathing motion". To quantify the extent of this motion, we computed the angle between two vectors, $\vec{m}$ and $\vec{n}$ drawn on the molecular frame of the protein and plotted its distribution. The vector from centre of mass (COM) of central $\beta$-sheet domain to COM of N terminal domain was termed as $\vec{m}$, and the vector from COM of central $\beta$-sheet domain to COM of C terminal domain was termed as $\vec{n}$ (Fig. $6 \mathrm{~b}$ inset). The angle subtended by these two vectors was computed using Eq. (1).

$$
\theta=\cos ^{-1}(\vec{m} \cdot \vec{n} /|\vec{m} \| \vec{n}|)
$$

From the angle distribution profile in Fig. 6b, it is apparent that TG-bound CETP is highly dynamic and can bend to an arch-like conformation with an angle as low as $118^{\circ}$ and subsequently stretched out to a more linear conformation with bending angle as large as $147^{\circ}$. For comparison, we have included the angle distribution profile of CE-bound CETP in Fig. 6b. As reported by us earlier, the CE-bound CETP spans its bending angle from about $131^{\circ}$ to $151^{\circ}$ with the mean resembling the crystal structure value of $139^{\circ}$ very well ${ }^{14,19}$. Clearly, TG-bound CETP explores a wider range of bending angles and the TG bound CETP is more bent than the CE bound CETP. This finding is consistent with the bulkier shape of TGs and their frequent inter-switching between different conformations as depicted in Fig. 5.

We also estimated the twisting-untwisting motion in CETP by computing the relative rotation of $\mathrm{N}$-terminal and C-terminal $\beta$-barrel domains with respect to the central $\beta$-domain. To quantify this, we constructed the axis of CETP as the vector connecting the COMs of $\mathrm{N}$ - and $\mathrm{C}$-terminals of central $\beta$-domain and subsequently defined two planes $P_{1}$ and $P_{2}$ with respect to this axis of CETP. The plane $P_{1}$ is constituted by the axis of CETP and COM of $\mathrm{N}$-terminal $\beta$-barrel domain, while plane $\mathrm{P}_{2}$ is constituted by the axis of CETP and COM of C-terminal $\beta$-barrel domain. The twisting angle $\left(\theta_{t}\right)$ was computed by measuring the angle between the normal vectors $\hat{n}_{1}$ and $\hat{n}_{2}$ of these two planes with the help of Eq. (1). Figure $6 \mathrm{c}$ shows the probability distribution of the twisting angle of CETP bound to both TG and CE. The figure depicts a Gaussian distribution with an average torsional twist of about $8^{\circ}$ in the CE-bound CETP crystal structure simulation. As this distribution indicates, CETP untwists (indicated by negative angle values), presumably to facilitate the transfer of CEs, and twists further (indicated by positive angle values) around the crystal structure value of $8^{\circ 19}$. The angle distribution in TG-bound CETP complex was found to be even wider with TG inducing more untwist to the CETP structure. This can be attributed to the lesser rigidity in the TG structure compared to $\mathrm{CE}$, with the latter having a rigid cholesterol moiety at the centre. Nevertheless, the wide range of bending and twisting angles indicates that CETP has sufficient structural plasticity and that can play a pivotal role in facilitating the transfer of the neutral lipids. The replica simulation on the TG-bound CETP system (replica simulation of system-I) also exhibits similar structural plasticity in CETP, as shown in Fig. S7.

At this juncture, it will be worth revisiting the convergence of our simulations. To do so, we divided the $200 \mathrm{~ns}-600 \mathrm{~ns}$ UA simulation data of system-I into four windows of $100 \mathrm{~ns}$ each, and computed the respective CETP bending angle distributions. The convergence of the results in Fig. S8 suggests that the CETP systems were well equilibrated and were stable in the timespan of the simulations.

Greater CETP-TG interactions explain slower transfer rate of TGs than CEs through CETP. Due to unavailability of any structural information of TG-bound CETP in literature, we attempted to validate our findings by correlating to the available functional data. Kinetic studies by several research groups have shown that CETP transfers CEs at a rate 2-8 fold faster than its rate of TG transfer ${ }^{23,24}$. To understand this differential lipid transfer activity of CETP, we calculated the number of protein-lipid contacts by counting the pairs of CETP and TG/CE sites that reside in close proximity with a chosen threshold distance of $5 \AA$. The results in Fig. 7 suggest that while TGs were involved in making about 2400 site-site contacts with CETP tunnel residues, CEs made only about 1800 contacts across the simulation time (also see Fig. S9 for the convergence of no. of contacts in replica TG-CETP system). Residue-level analysis on the time-averaged structures of CE and TG bound CETP also showed similar trend, even though both lipids showed multiple common interactions with CETP tunnel residues. The interactions of CETP tunnel residues with $\mathrm{N}$-terminal CE $\left(\mathrm{CE}_{\mathrm{N}}\right)$ and $\mathrm{N}$-terminal TG $\left(\mathrm{TG}_{\mathrm{N}}\right)$ are included in Fig. 7. Unsurprisingly, both $\mathrm{CE}_{\mathrm{N}}$ and $\mathrm{TG}_{\mathrm{N}}$ have many common interactions with CETP residues, e.g. ILE11, CYS13, and ILE15 in strand-S1; VAL30, ILE31, ALA34, and PHE35 in helix-A; LEU123 and ILE125 in strand-S5; ILE183, ILE187, MET194, VAL198, and LEU206 in helix-B; ILE215 in strand-S7; HIS232 in strand-S8; PHE263 and PHE265 in strand-S1'; LEU273 in helix- $\mathrm{A}^{\prime}$ interact with both $\mathrm{CE}_{\mathrm{N}}$ and $\mathrm{TG}_{\mathrm{N}}$. Apart from the CETP residues, plug-in phospholipids (PLs) also participate in similar interactions with both $\mathrm{CE}_{\mathrm{N}}$ and $\mathrm{TG}_{\mathrm{N}}$. The naming of the CETP secondary structural elements, e.g. strand-S1, helix-A, strand-S1' etc. is adopted from the CETP crystal structure article, ref. 19.

However, $\mathrm{TG}_{\mathrm{N}}$ has lost hydrophobic interactions with tunnel residues: ILE82 and VAL84 in strand-S4; THR127 and LEU129 in strand-S5; THR138 in strand-S6; CYS184 and ILE190 in helix-B; VAL213 in strand-S7; LEU228 and SER230 in strand-S8; VAL269 and PHE270 in helix-A'; LEU472 in helix-X. In compensation, $\mathrm{TG}_{\mathrm{N}}$ gains new contacts with residues LEU23 in helix-A; ILE84 in strand-S4; GLN124 in strand-S5; VAL136, CYS143, TYR144, and LEU145 in strand-S6; SER191, ALA195, and ALA202 in helix-B; PHE236 in strand-S8; LEU261 in strand-S1'; MET433, VAL438, and PHE441 helix-B'; ILE443 and PRO446 in strand-S7'; LEU457, MET459, and PHE461 in strand-S8'; and the C-terminal TG, $\mathrm{TG}_{\mathrm{C}}$ (compare Fig. $7 \mathrm{~b}$ and c).

Apart from the above-mentioned hydrophobic contacts, $\mathrm{TG}_{\mathrm{N}}$ also involved in $\mathrm{H}$-bonding interaction with CETP helix-B residue, GLN199 as indicated in Fig. 7b. Overall, there was a net gain of 8 residue-level contacts for 

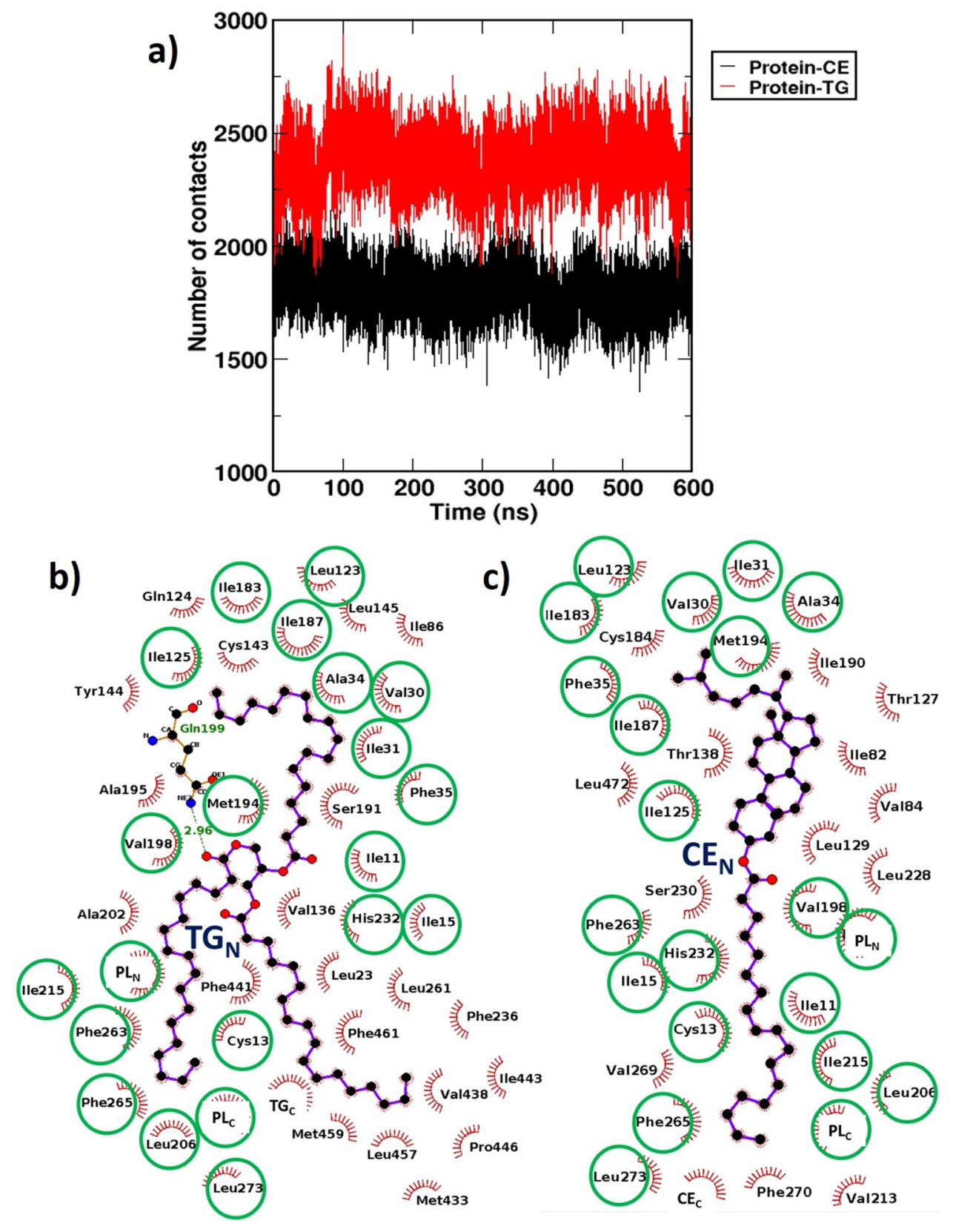

Figure 7. (a) Time evolution of the number of hydrophobic contacts formed by TG (red) and CE (black) with CETP. Protein-lipid contacts were estimated by counting the pairs of CETP and TG/CE sites that reside in close proximity with a chosen threshold distance of $5 \AA$. The interactions of CETP tunnel residues with (b) $\mathrm{N}$-terminal TG in TG-CETP complex and (c) N-terminal CE in CE-CETP complex are shown for comparison. TG and CE are shown in ball-and-stick representation and protein residues involved in hydrophobic interactions are shown by red spikes. H-bonding interactions between TG and CETP residue Gln 199 is shown by green dotted lines. Green circles denote the common interactions in both complexes.

$\mathrm{TG}_{\mathrm{N}}$ over the native 43 contacts that $\mathrm{CE}_{\mathrm{N}}$ makes in CETP tunnel, accounting for a net gain of $18.6 \%$ contacts for $\mathrm{TG}_{\mathrm{N}}$. Similarly, $\mathrm{TG}_{\mathrm{C}}$ also exhibited additional interactions with CETP tunnel lining residues as shown in Fig. S10 $\left(\mathrm{TG}_{\mathrm{C}}\right.$ and $\mathrm{CE}_{\mathrm{C}}$ exhibited 48 and 38 residue-level contacts, respectively), which account for a net increase of $20.8 \%$ residue-level contacts over $\mathrm{CE}_{\mathrm{C}}$. This is interesting, since the TG molecules could experience a larger friction when traversing through the CETP tunnel due to afore-discussed additional hydrophobic and hydrogen bonding interactions. This, along with more bent conformation of CETP, could limit the transfer rate of TG and explain its slower kinetics compared to CE.

Identified CETP residues that favor TG/CE binding match well with mutagenesis data. To further validate our proposed TG-bound CETP structure, we identified key CETP residues that interact with bound TGs favorably and compare those with the CETP residues that are reported to modulate the TG transfer activity 

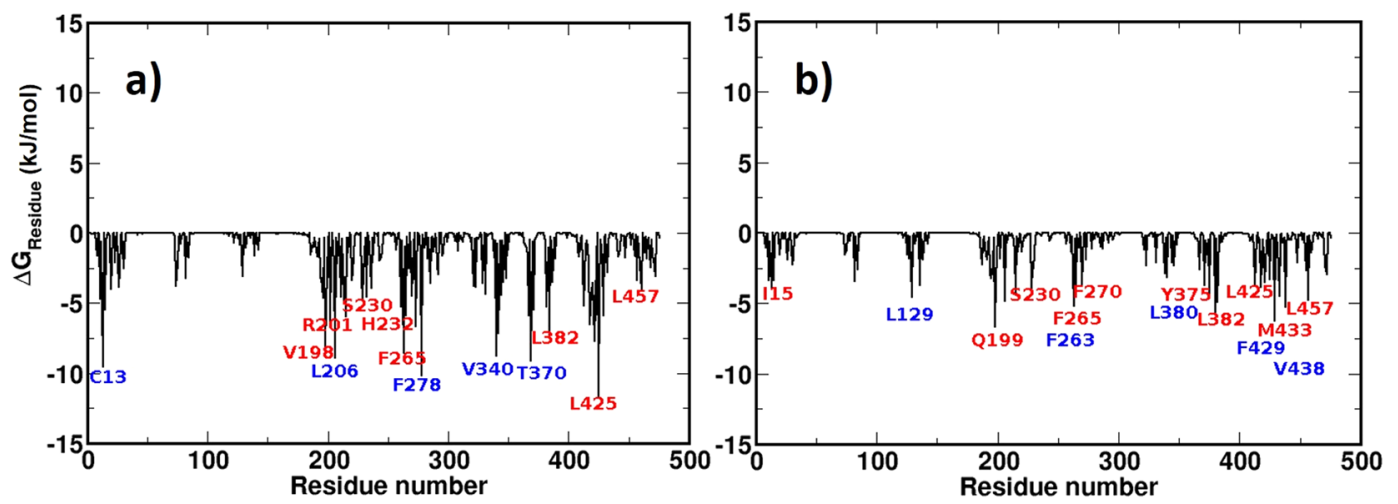

Figure 8. Gibbs free energy of binding of individual CETP residues with (a) TG molecules in CETP-TG complex and (b) CE molecules in CETP-CE complex. Residues that exhibited diminished lipid transfer activity upon mutations, in recent mutagenesis studies, are labeled red and the other important residues are labeled blue.

from recent mutagenesis studies. For this, we first calculated the free energy of binding of TG in CETP core and subsequently assess the energetic contribution of individual CETP residues in TG binding. For comparison, the same calculation was performed on the control CE bound CETP complex. Free energy calculations were performed by GROMACS utility program, g_mmpbsa that uses the molecular mechanics Poisson Boltzmann surface area method ${ }^{25}$. The detailed methodology for evaluating the free energy of ligand binding is presented by us and many other authors earlier ${ }^{14,26}$. The estimated free energy values for TG and CE binding were $-433.12 \mathrm{~kJ} / \mathrm{mol}$ and $-239.32 \mathrm{~kJ} / \mathrm{mol}$, respectively, suggesting a much stronger binding of TGs in CETP, which again corroborates very well with TG's 2-8 fold slower transfer rate through CETP hydrophobic tunnel than CEs.

To obtain the energetic contribution of individual CETP residues, we decomposed the total Gibbs free energy into residue level. A set of 10,000 conformations from the last 50 ns of TG-bound CETP MD trajectory was analyzed and the residues with significant contributions are presented in Fig. 8a. To list out the significantly contributing residues, we have chosen a free energy cut-off value of $-4 \mathrm{~kJ} / \mathrm{mol}$. As Fig. 8 a shows, TGs have strong binding affinity for the central $\beta$-domain residues CYS13, SER230, HIS232, PHE265; helix-B residues VAL198, ARG201, LEU206; helix-A' residue PHE278; strand-S4' residue VAL340; strand-S5' residue THR370; strand-S6' residue LEU382; helix-B' residue LEU425; and strand-S8' residue LEU457. Interestingly, this free energy results corroborate well with the contact analysis data presented above. Most of the strongly interacting CETP residues (e.g. CYS13, VAL198, LEU206, SER230, HIS232, PHE265, VAL340, LEU382, and LEU457) were also found in the CETP-TG contact analysis (Figs 8a and S10a). More importantly, a subset of our identified residues with significant affinity for TGs has recently been reported to be crucial in TG transfer through CETP ${ }^{19}$. For example, the mutants R201S and V198W have abolished the TG-transfer activity of CETP up to 90\%. The mutant S230A has exhibited nearly $80 \%$ decrease in TG-transfer activity. Similarly, H232A, L425W, and L457W have respectively reported nearly $60 \%, 40 \%$, and $70 \%$ loss in TG-transfer activity. Furthermore, the mutants F265R and $\mathrm{L} 382 \mathrm{~W}$ were characterized for their significant role in the misfolding of CETP and subsequent failure in CETP secretion $^{19}$.

In similar context, we have also analyzed the energetic contribution of individual CETP residues interacting with CEs and the results are presented in Fig. $8 \mathrm{~b}$. As shown, CEs have strong binding with central $\beta$-domain residues ILE15, SER230, PHE263, PHE265, PHE270; helix-B residue GLN199; strand-S5 residue LEU129; strand-S5' residue TYR375; strand-S6' residues LEU380, LEU382; helix B' residues LEU425, PHE429, MET433, VAL438; and strand-S8' residue LEU457. Most of these interacting residues also match well with the CETP-CE contact analysis data, e.g. ILE15, LEU129, SER230, PHE263, PHE265, PHE270, TYR375, LEU380, LEU382, LEU425, PHE429, MET433, VAL438, and LEU457 (Figs 7c and S10b). Moreover, these data from free energy analysis corroborates very well with the available mutagenesis data. For example, the mutants Q199A, S230A, L425W, and M433W have exhibited nearly 75\%,50\%,50\%, and 40\% loss in CE-transfer activity of CETP, respectively. Similarly, I15W and L457W abolished the CE-transfer activity up to $60 \%$.

We believe that the 2-8 fold slower transfer rate of TGs through CETP could be a consequence of this differential interaction of TG and CE with CETP. TG molecules experience a larger friction while traversing through CETP tunnel due to additional hydrophobic and hydrogen bonding interactions. Moreover, the strength of binding of TGs in CETP hydrophobic tunnel is significantly higher than the CE binding. These, along with more bent conformation of CETP, could limit the transfer rate of TGs and explain its slower kinetics compared to CEs through CETP tunnel. Such a good structure-function correlation validate our proposed structure of TG bound CETP.

\section{Conclusions}

CETP transfers CEs from HDL to LDL/VLDL with the complementary transfer of TGs from LDL/VLDL to HDL. Interestingly, both the solved crystal structures of apo and inhibitor bound CETP are in CE-bound state. However, recent mutational studies have shown that CETP is also an active TG transporter, since the protein looses significant CE transfer activity upon the inhibition of TG transfer by active mutations in the hydrophobic 
tunnel. A more direct evidence of the existence of TG bound CETP came from radiolabeled assays. Unfortunately, till date, no structural information of TG-bound CETP is available. This has motivated us to explore the TG conformations in CETP through large-scale MD simulations. Our results from coarse-grained simulations show that, irrespective of the initial conformations, the pair of docked TGs in CETP core tunnel converges to "parallel N-N" orientation, where both TGs orient head-to-legs from N- to C-terminal of CETP. This finding can be justified by the facts that TGs enter into CETP tunnel predominantly through the C-terminal end ${ }^{13}$, and since entering the narrow tunnel by inserting only one of the three identical oleate chains would require less work, TGs enter CETP in head-to-leg conformation with the single oleate chain (head) pointing toward the N-domain and the other two chains (legs) pointing toward C-domain. Moreover, since the tunnel in N-domain of CETP is narrower, after entering in the head-to-legs conformation, TGs remains stable in such form for majority of the time.

After finding the "parallel N-N" as the major conformation of the bound TGs in CETP, we subsequently checked their influence on the structural stability of CETP and also compared the obtained TG-bound CETP structure with the available CE-bound CETP crystal structure. In this context, our results from all-atom MD simulations show that in spite of significant changes in TG conformations in the core of CETP, the protein maintained its secondary and tertiary structures with the bending and twisting angle distributions mimicking the CE-bound CETP crystal structure values. We further attempted to validate our findings by explaining the reported 2-8 fold slower kinetics of TG through CETP, where we have shown that TGs bind very strongly and make $20 \%$ larger contacts with CETP tunnel than CEs. We speculate that this along with the more bent CETP conformation in this complex make TG passage slower through CETP. Findings are strengthened further by showing that the identified CETP residues facilitating TG/CE binding are the ones that affected TG/CE transfer when mutated in recent mutagenesis studies. It is to be noted that the proposed TG-bound CETP structure is stable when CETP is in solution and not bound to the lipoprotein particles. When CETP binds to HDL/LDL, it is possible that TGs move out of CETP tunnel and transfer to the HDL/LDL lipid monolayers. Work in this direction is in progress in our laboratory. We believe that this study will help the CETP research community to understand its TG/CE transfer activity more comprehensively. Further, the present study would pave way to design new experiments on TG-bound CETP complexes.

\section{Materials and Methods}

The coordinate file of CETP with PDB ID 2OBD was downloaded from the RCSB Protein Data Bank ${ }^{19}$. Four $\mathrm{N}$-terminal missing residues, ${ }^{1} \mathrm{~A}-\mathrm{S}-\mathrm{K}-\mathrm{G}^{4}$ were incorporated using Modeller $9 \mathrm{v} 13$ tool $^{27}$ and the mutations induced in CETP to promote the formation of crystal, viz. C1A, N88D, C131A, N240D, and N341D were reverted to original residues. Subsequently, to prepare the TG-bound CETP initial structure, the bound neutral lipids CEs were stripped out from the CETP core tunnel, while retaining both the charged phospholipids in their crystal structure positions. Two triglyceride (TG) molecules were docked manually into the CETP tunnel in place of two CEs. We chose triolein molecules (Fig. 1a) to represent the TGs in CETP, based on the proposition in CE-bound CETP crystal structure report ${ }^{19}$.

The prepared systems were subjected to united-atom (UA) and coarse-grained (CG) molecular dynamics simulations for long enough time to understand the structure and dynamics of TG bound CETP complexes. The united-atom simulations were carried out using GROMOS53A6 forcefield parameters for protein ${ }^{28}$ and Berger lipid parameters ${ }^{29}$ for lipids, where the aliphatic (non-polar) hydrogens with attached carbons were defined as single reaction center while all polar hydrogens were treated explicitly. The forcefield parameters for triolein molecules were obtained from the reported liquid phase TG simulation system ${ }^{22}$. GROMACS-4.5.5 simulation package was used to execute all the simulations ${ }^{30}$. The protonation states of histidine residues in CETP were identified and incorporated using WHATIF program ${ }^{31}$. Initially, the protein-lipid complexes were briefly minimized for 1000 steps using the steepest descent algorithm and another 1000 steps using conjugate gradient algorithm. Then the structures were solvated with explicit water in cubic periodic box. SPC water model was chosen to describe the water molecules. The primary TG-bound CETP system was solvated with 102,900 water molecules at a salt concentration of $0.15 \mathrm{M}$ by including $297 \mathrm{Na}^{+}$and $290 \mathrm{Cl}^{-}$ions. The total size of this system was about 350,000 atoms. The solvated systems were subjected to extensive energy minimization and subsequent heating to $310 \mathrm{~K}$ in a canonical ensemble using Nose-Hoover thermostat with a coupling constant of 1.0 ps. Next isothermal-isobaric conditions were adopted at $310 \mathrm{~K}$ and 1 bar to adjust the solvent density. The pressure was set to $1 \mathrm{bar}$ using the Parrinello-Rahman barostat with isotropic pressure coupling of coupling constant 0.1 ps. The van der Waals interactions were cut-off at $1.0 \mathrm{~nm}$. Electrostatic interactions were defined by the particle-Mesh Ewald sum with a real space cut-off value at $1.0 \mathrm{~nm}$. LINCS algorithm was used to constrain all bonds involving hydrogen atoms. The systems were equilibrated for $10 \mathrm{~ns}$ in UA simulations with a time step of $2 \mathrm{fs}$ in NPT ensemble. Finally the production run was performed for 400-600 ns.

Apart from the united atom simulations, we have also performed coarse-grained (CG) simulations on the TG-bound CETP systems to observe the large-scale conformational changes. CG simulations were carried out by employing ElNeDyn forcefeild on CETP through the integration of elastic network model ${ }^{32}$ with Martini forcefield $^{33}$. The elastic network model was employed to retain the secondary structures of CETP during the CG simulations. The CG bead mapping and forcefield parameters for TG were adopted from the work of Vattulainen and coworkers ${ }^{34}$. Briefly, the bead mapping of TG molecules was as follows: each TG is coarse grained based on a four-to-one mapping, i.e., every four heavy atoms are represented by a single interaction site. As Fig. $1 \mathrm{~b}$ shows, the central glycerol moiety is modeled as GLY bead and the adjoining three carboxyl moieties are represented as sites of intermediate hydrophilicity (ES1, ES2, and ES3). Each of the three oleoyl chains is represented by four beads three of apolar type and a slightly more polar one to account for the double bond (D2A, D2B, and D2C). The obtained coarse-grained TG was docked into the hydrophobic tunnel of CETP and simulated in CG water model. The primary TG-bound CETP system was solvated in CG water beads and subsequently neutralized by adding $11 \mathrm{Na}^{+}$ions. Temperature was maintained at $310 \mathrm{~K}$ using velocity rescale thermostat with a coupling constant of 
$1.0 \mathrm{ps}$, and pressure was maintained at 1 bar using Parrinello-Rahman barostat with isotropic pressure coupling. All the electrostatic and van der Waals interactions were cut-off at $1.2 \mathrm{~nm}$. The production run was performed with a time step of $20 \mathrm{fs}$. All the structural figures were rendered using $\mathrm{VMD}^{35}$ and $\mathrm{PyMOL}^{36}$.

\section{References}

1. Barter, P. et al. HDL Cholesterol, Very Low Levels of LDL Cholesterol, and Cardiovascular Events. N. Engl. J. Med. 357, 1301-1310 (2007).

2. Di Angelantonio, E. et al. Major lipids, apolipoproteins, and risk of vascular disease. JAMA - J. Am. Med. Assoc 302, 1993-2000 (2009).

3. Assmann, G., Cullen, P. \& Schulte, H. Simple Scoring Scheme for Calculating the Risk of Acute Coronary Events Based on the 10Year Follow-Up of the Prospective Cardiovascular Münster (PROCAM) Study. Circ 105, 310-315 (2002).

4. Rosenson, R. S. et al. Cholesterol Efflux and Atheroprotection: Advancing the Concept of Reverse Cholesterol Transport. Circ 125, 1905-1919 (2012).

5. Voyiaziakis, E. et al. ApoA-I deficiency causes both hypertriglyceridemia and increased atherosclerosis in human apoB transgenic mice. J. Lipid Res. 39, 313-321 (1998).

6. Barter, P. J. et al. Cholesteryl ester transfer protein: A novel target for raising HDL and inhibiting atherosclerosis. Arterioscler. Thromb. Vasc. Biol 23, 160-167 (2003).

7. Barkowski, R. S. \& Frishman, W. H. HDL metabolism and CETP inhibition. Cardiol. Rev. 16, 154-162 (2008)

8. Nissen, S. E. et al. Effect of Torcetrapib on the Progression of Coronary Atherosclerosis. N. Engl. J. Med. 356, 1304-1316 (2007).

9. Schwartz, G. G. et al. Effects of dalcetrapib in patients with a recent acute coronary syndrome. N. Engl. J. Med. 367, 2089-2099 (2012).

10. Gotto, A. M. \& Moon, J. E. Safety of inhibition of cholesteryl ester transfer protein with anacetrapib: The DEFINE study. Expert Rev. Cardiovasc. Ther. 10, 955-963 (2012).

11. Qiao, J. X. et al. Triphenylethanamine Derivatives as Cholesteryl Ester Transfer Protein Inhibitors: Discovery of N-[(1R)-1-(3Cyclopropoxy-4-fluorophenyl)-1-[3-fluoro-5-(1,1,2,2-tetrafluoroethoxy)phenyl]-2-phenylethyl]-4-fluoro-3-(trifluoromethyl) benzamide (BMS-795311). J. Med. Chem. 58, 9010-9026 (2015).

12. Chirasani, V. R., Sankar, R. \& Senapati, S. Mechanism of Inhibition of Cholesteryl Ester Transfer Protein by Small Molecule Inhibitors. J. Phys. Chem. B 120, 8254-8263 (2016).

13. Zhang, L. et al. Structural basis of transfer between lipoproteins by cholesteryl ester transfer protein. Nat. Chem. Biol. 8, 342-349 (2012).

14. Chirasani, V. R., Revanasiddappa, P. D. \& Senapati, S. Structural Plasticity of Cholesteryl Ester Transfer Protein Assists the Lipid Transfer Activity. J. Biol. Chem. 291, 19462-19473 (2016).

15. Lei, D. et al. Insights into the Tunnel Mechanism of Cholesteryl Ester Transfer Protein through All-atom Molecular Dynamics Simulations. J. Biol. Chem. 291, 14034-14044 (2016).

16. Zhang, M. et al. HDL surface lipids mediate CETP binding as revealed by electron microscopy and molecular dynamics simulation. Sci. Rep 5, 8741 (2015).

17. Koivuniemi, A. et al. Lipid Exchange Mechanism of the Cholesteryl Ester Transfer Protein Clarified by Atomistic and Coarsegrained Simulations. PLoS Comput Biol 8, e1002299 (2012).

18. García-González, V. et al. Key structural arrangements at the C-terminus domain of CETP suggest a potential mechanism for lipidtransfer activity. J. Struct. Biol. 186, 19-27 (2014).

19. Qiu, X. et al. Crystal structure of cholesteryl ester transfer protein reveals a long tunnel and four bound lipid molecules. Nat. Struct. Mol. Biol. 14, 106-113 (2007).

20. Liu, S. et al. Crystal structures of cholesteryl ester transfer protein in complex with inhibitors. J. Biol. Chem. 287, 37321-37329 (2012).

21. Swenson, T. L. et al. Plasma cholesteryl ester transfer protein has binding sites for neutral lipids and phospholipids. J. Biol. Chem. 263, 5150-5157 (1988).

22. Hall, A., Repakova, J. \& Vattulainen, I. Modeling of the Triglyceride-Rich Core in Lipoprotein Particles. J. Phys. Chem. B 112, 13772-13782 (2008).

23. Connolly, D. T. et al. Physical and kinetic characterization of recombinant human cholesteryl ester transfer protein. Biochem. J. 320, 39-47 (1996).

24. Ohnishi, T., Oikawa, K., Kay, C. M. \& Yokoyama, S. Modulation of substrate selectivity in plasma lipid transfer protein reaction over structural variation of lipid particle. Biochim. Biophys. Acta - Lipids Lipid Metab 1254, 117-126 (1995).

25. Kumari, R., Kumar, R. \& Lynn, A. g-mmpbsa- A GROMACS tool for high-throughput MM-PBSA calculations. J. Chem. Inf. Model. 54, 1951-1962 (2014)

26. Baker, N. A., Sept, D., Joseph, S., Holst, M. J. \& McCammon, J. A. Electrostatics of nanosystems: application to microtubules and the ribosome. Proc. Natl. Acad. Sci. USA 98, 10037-10041 (2001).

27. Webb, B. \& Sali, A. Comparative protein structure modeling using Modeller. Curr. Protoc. Bioinforma 47, 5-6 (2014).

28. Schuler, L. D., Daura, X. \& van Gunsteren, W. F. An improved GROMOS96 force field for aliphatic hydrocarbons in the condensed phase. J. Comput. Chem. 22, 1205-1218 (2001).

29. Berger, O., Edholm, O. \& Jahnig, F. Molecular Dynamics Simulations of a Fluid Bilayer of Dipalmitoylphosphatidylcholine at Full Hydration, Constant Pressure, and Constant Temperature. Biophys. J. 72, 2002-2013 (1997).

30. Hess, B., Kutzner, C., van der Spoel, D. \& Lindahl, E. GROMACS 4: Algorithms for Highly Efficient, Load-Balanced, and Scalable Molecular Simulation. J. Chem. Theory Comput. 4, 435-447 (2008).

31. Vriend, G. WHAT IF: A molecular modeling and drug design program. J. Mol. Graph. 8, 52-56 (1990)

32. Periole, X., Cavalli, M., Marrink, S.-J. \& Ceruso, M. A. Combining an Elastic Network With a Coarse-Grained Molecular Force Field: Structure, Dynamics, and Intermolecular Recognition. J. Chem. Theory Comput. 5, 2531-2543 (2009).

33. Marrink, S. J., Risselada, H. J., Yefimov, S., Tieleman, D. P. \& Vries, A. H. De The MARTINI Force Field: Coarse Grained Model for Biomolecular Simulations The MARTINI Force Field: Coarse Grained Model for Biomolecular Simulations. J. Phys. Chem. B 111, 7812-7824 (2007).

34. Vuorela, T. et al. Role of Lipids in Spheroidal High Density Lipoproteins. PLoS Comput Biol 6, e1000964 (2010)

35. Humphrey, W., Dalke, A. \& Schulten, K. VMD: Visual molecular dynamics. J. Mol. Graph. 14, 33-38 (1996).

36. The PyMOL Molecular Graphics System, Version 1.5.0.4 Schrödinger, LLC.

\section{Acknowledgements}

We thank Prof. I. Vattulainen for providing us the united-atom force field parameters for TG. We also thank IIT Madras for funding. 


\section{Author Contributions}

Conceived and designed the experiments: V.R.C., S.S. Performed the experiments: V.R.C. Analyzed the data: V.R.C., S.S. Contributed reagents/materials/analysis tools: S.S. Wrote the paper: V.R.C., S.S.

\section{Additional Information}

Supplementary information accompanies this paper at doi:10.1038/s41598-017-05449-z

Competing Interests: The authors declare that they have no competing interests.

Publisher's note: Springer Nature remains neutral with regard to jurisdictional claims in published maps and institutional affiliations.

(1) Open Access This article is licensed under a Creative Commons Attribution 4.0 International License, which permits use, sharing, adaptation, distribution and reproduction in any medium or format, as long as you give appropriate credit to the original author(s) and the source, provide a link to the Creative Commons license, and indicate if changes were made. The images or other third party material in this article are included in the article's Creative Commons license, unless indicated otherwise in a credit line to the material. If material is not included in the article's Creative Commons license and your intended use is not permitted by statutory regulation or exceeds the permitted use, you will need to obtain permission directly from the copyright holder. To view a copy of this license, visit http://creativecommons.org/licenses/by/4.0/.

(C) The Author(s) 2017 